\title{
Erratum to: Comparison of blood glucose levels and allergic responses on treatment with six wheat cultivars
}

\author{
Hyeri Lee ${ }^{1} \cdot$ Min Hee Hwang ${ }^{1}$ Miju Cho ${ }^{1}$ Dong Gun Lee ${ }^{1} \cdot$ \\ Eun Byeol Go ${ }^{1}$ - Young-Keun Cheong ${ }^{2}$ Chon-Sik Kang ${ }^{2}$. \\ Nam Taek Lee $^{3} \cdot$ Namhyun Chung ${ }^{1}$
}

Published online: 14 February 2017

(C) The Korean Society for Applied Biological Chemistry 2017

\section{Erratum to: Appl Biol Chem \\ DOI 10.1007/s13765-016-0241-0}

Unfortunately, there are errors in Figs. 1 and 2 in the originally published article. Correct Figs. 1 and 2 are shown in this erratum.

The online version of the original article can be found under doi:10.1007/s13765-016-0241-0.

Nam Taek Lee

ntlee@korea.ac.kr

$\bowtie$ Namhyun Chung

nchung@korea.ac.kr

1 Department of Biosystems Engineering, College of Life Sciences and Biotechnology, Korea University, Seoul 02841, Republic of Korea

2 National Institute of Crop Science, RDA, Wanju Gun 55365, Republic of Korea

3 Functional Food Research Center, College of Life Sciences and Biotechnology, Korea University, Seoul 02841, Republic of Korea 

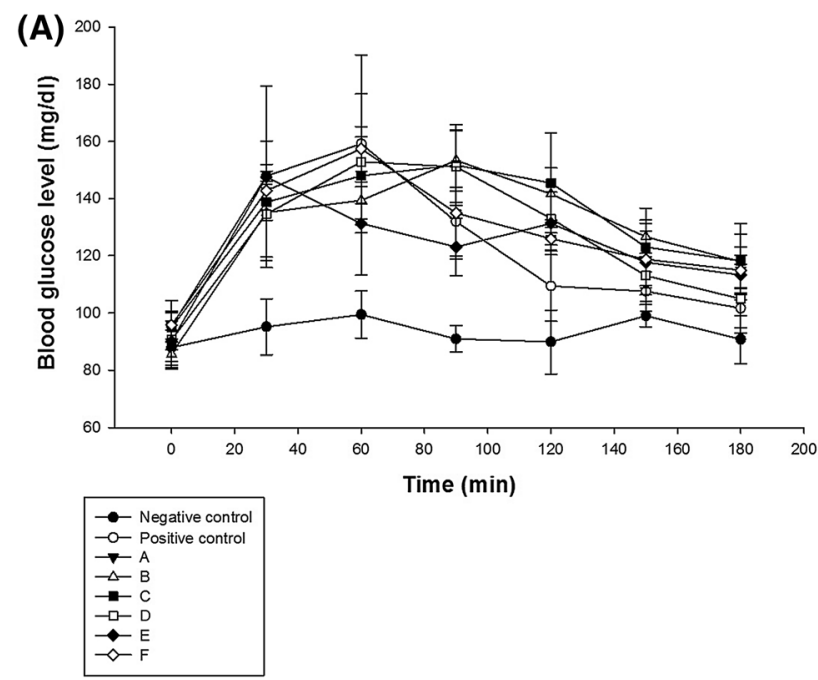

Fig. 1 Changes in blood glucose levels after administration of wheat flours from six wheat cultivars. $N C$ negative control and $P C$ positive control. The wheat cultivars: $A$ HRW, $B$ AH, $C$ IGSW, $D$ GSW,

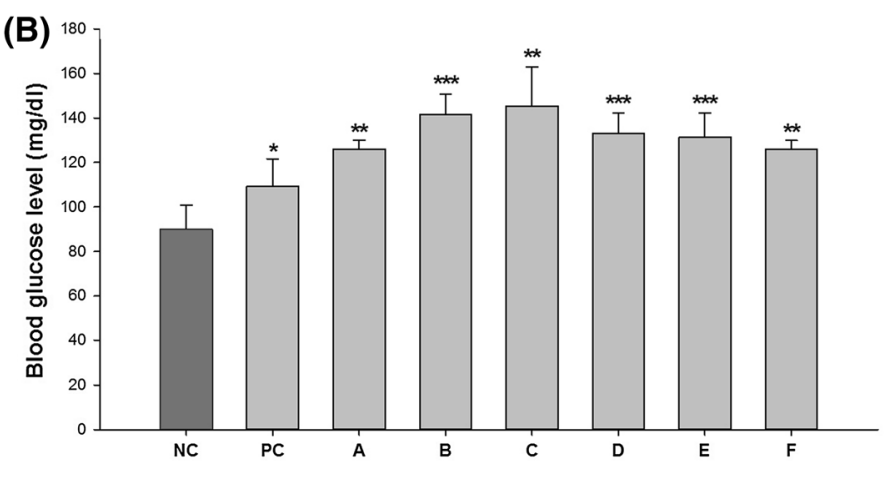

$E$ ASW, $F$ GKW. Data are represented as mean $\pm \mathrm{SD}(n=5)$ at every $30 \mathrm{~min}(\mathbf{A})$. The blood glucose levels at $120 \mathrm{~min}$ after wheat flour administration. $* p<0.05, * * p<0.01, * * * p<0.00$ ( $t$ test) (B)

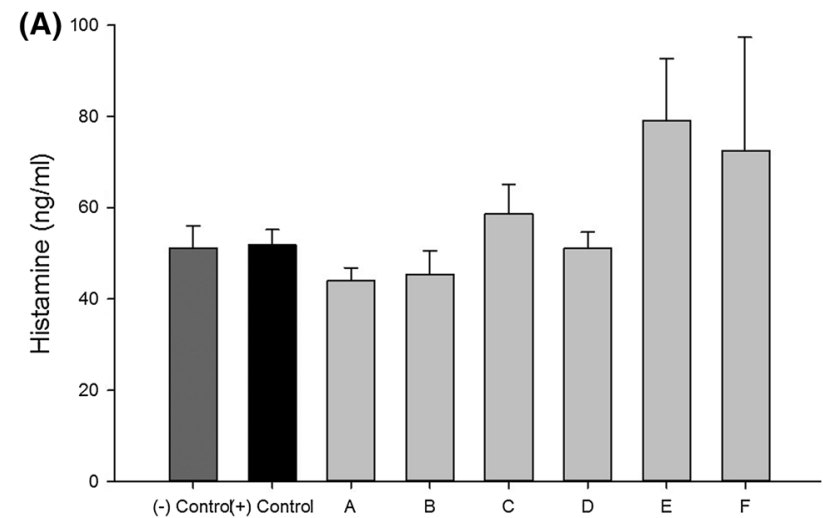

Fig. 2 Allergic responses observed as secretion levels of histamine and $\mathrm{IgE}$ in $\mathrm{C} 3 \mathrm{H} / \mathrm{HeJ}$ mice after administration of wheat flour from six wheat cultivars. Variation in the secretion levels of histamine (A) and $\operatorname{IgE}$ (B) according to wheat cultivars is shown. Data are the

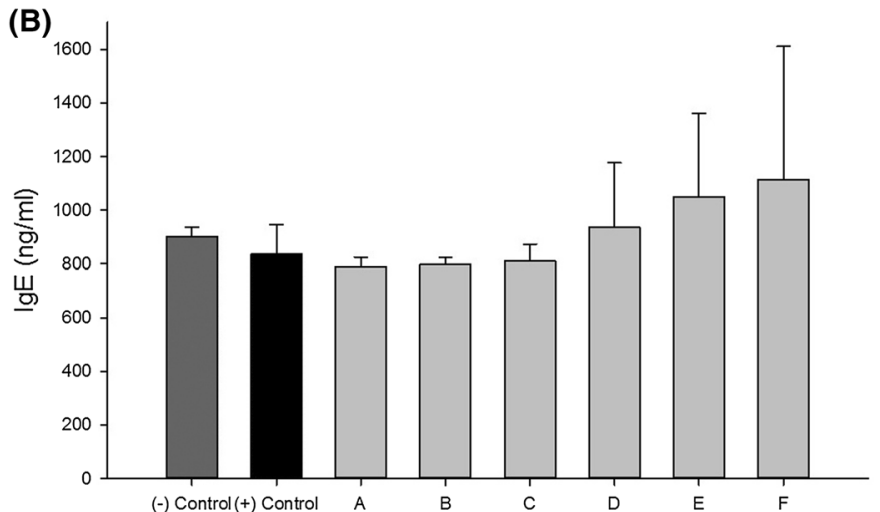

mean $\pm \mathrm{SD}$ from triplicate experiments. $N C$ negative control and $P C$ positive control. The wheat cultivars: $A$ HRW, $B$ AH, $C$ IGSW, $D$ GSW, $E$ ASW, and $F$ GKW. Data are the mean \pm SD from triplicate experiments 